\title{
RESULTADOS DE DATACIONES RADIOCARBONICAS DEL SITIO ARQUEOLOGICO ANGIE
}

Sagrario Balladares, Grethel K. Gaitán, Leonardo Lechado

\begin{abstract}
Nota explicativa
Como parte de convenios de colaboración e intercambio, un equipo de arqueólogos investigadores pertenecientes al Centro de Investigación y Documentación de la Costa Atlántica, de la Bluefields Indian \& Caribbean University, BICU-CIDCA y la Facultad de Humanidades y Ciencias Jurídicas de la UNAN Managua, realizaron trabajos de investigación en la región del Caribe de gran relevancia académica y científica.

El informe que a continuación se publica recoge las interpretaciones realizadas por el equipo sobre las dataciones radio carbónicas aplicadas a recientes hallazgos arqueológicos en la Costa Caribe nicaragüense. Es un primer estudio al cual le seguirán otros en esa zona, los cuales prometen resultados de gran trascendencia para entendimiento de la evolución humana y su entorno cultural.
\end{abstract}

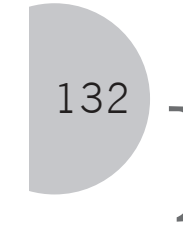

\section{Introducción}

L os resultados de dataciones radio carbónicas que a continuación se del proceso de investigación arqueológica que se viene desarrollando en el Territorio Rama-Kriol, desde el año 2012 por BICU-CIDCA. Proyecto coordinado por el Licenciado Donald H. Byers con la colaboración científico técnica de la UNAN, Managua.

Estas dataciones realizadas en los laboratorios Beta Analityc Inc de Miami, USA, fueron posibles gracias al financiamiento recibido por la Embajada de Estados Unidos de América en Nicaragua.

\section{Antecedentes}

En la década de los 70s, el arqueólogo nicaragüense Jorge Espinoza Estrada, realizó excavaciones arqueológicas en uno de los concheros existentes en la comunidad de Monkey Point, en el litoral del Caribe nicaragüense. Este conchero fue denominado como Angie con Código, N-RS-Mp-001en el Registro Nacional de Bienes Arqueológicos.

Según el investigador estadounidense Richard Magnus (1974) quien citó a J. Espinoza (1974), el sitio en aquel momento, presentaba una antigüedad aproximada de 7600 años. No obstante, durante todos esos años se hizo imposible contar con algún tipo de materialidad o documentos que certificara estos datos.

En noviembre del2013, dentro del marco del proyecto "Fortalecimiento del patrimonio cultural arqueológico en el territorio Rama y Kriol en la región Autónoma del Atlántico Sur, RAAS, Nicaragua", un equipo de investigadores conformado por arqueólogos de la BICU-CIDCA y de la UNAN-Managua, visitaron la región donde se emplaza este conchero con la finalidad de verificar in situ la existencia y el estado de conservación del mismo. En

Sagrario Balladares, Grethel K. Gaitán, Leonardo Lechado 
esa ocasión corroboraron la presencia, no solo de uno, sino de tres concheros más.

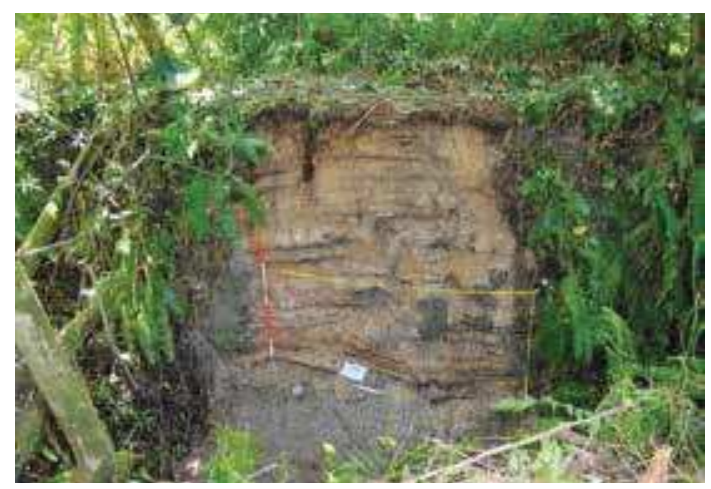

Limpieza de perfil del conchero Angie, en Monkey Point. 2013. Nótese parcialmente la cata de sondeo realizada por J Espinoza en los años 70 del siglo pasado.

\section{El trabajo de campo y su proceso de datación}

El sitio arqueológico Angie tiene la particularidad de presentar una antigua trinchera arqueológica de $18.20 \mathrm{~m}$ de largo en el eje N-S; de $5.50 \mathrm{~m}$ en la parte más ancha en el eje E-O y de $5 \mathrm{~m}$ en la parte más profunda situada en el sector N. Dentro de esta trinchera, se seleccionó un pequeño espacio de $3 \mathrm{~m}$ de ancho por $3.55 \mathrm{~m}$ de profundidad localizado en el perfil oeste y hacia el extremo norte de la trinchera de donde se extrajeron muestras para obtener una caracterización del sitio.

La acción de extracción representó en ese momento, una oportunidad única si se tiene en cuenta los altos costos que representa penetrar en a la zona. Según información de habitante de la localidad donde se encuentra ubicado el sitio, muchos antiguos trabajadores o colaboradores en las labores de campo de Jorge Espinoza, el conchero media $7 \mathrm{~m}$ de profundidad aproximadamente.

El trabajo de campo en el perfil consistió en: limpieza de sitio, documentación

Sagrario Balladares,

Grethel K. Gaitán,

Leonardo Lechado técnica, gráfica, fotográfica y planimétrica, extracción de muestras del perfil en diversos estratos de la secuencia estratigráfica observada. Además se extrajeron muestras de carbones y conchas con el fin de establecer el tiempo de origen de estos por medio del método de datación de C-14 con miras a corroborar las fechas propuestas para este sitio.

Una vez limpio el perfil y mediante el análisis del mismo, se hizo un hallazgo importante el cual fue extraído. Este consistió en la identificación de una osamenta humana orientada norte-sur, la cual se encontraba depositada en una pequeña fosa en posición decúbito dorsal, con las extremidades inferiores flexionadas y las extremidades superiores extendidas, y con la parte frontal del cráneo en posición vertical y en dirección hacia el Este. Por debajo de la osamenta se identificaron pequeños clastos basálticos (menores de $10 \mathrm{~cm}$ ) depositados como base del entierro, los que a su vez se apoyaban en un nivel de sedimentos con carbones cuyo espesor mayor no sobrepasaba los $10 \mathrm{~cm}$.

Las condiciones de conservación que presentaba la osamenta no eran las óptimas debido al paso del tiempo y al proceso de fosilización al que estaba sometido. Debido a eso, las dos muestras recolectadas de ella para dataciones radio carbónicas fueron rechazadas por el Laboratorio BETA, otra razón era que le faltaba colágeno en dientes y huesos largos.

No obstante, se le realizaron análisis físicos para determinar tamaño, sexo, edad y posibles deformaciones. Estos análisis fueron realizados en BICU-CIDCA el pasado mes de marzo por la Dra. Mirjana Roksandic, antropóloga biológica de la Universidad de Winnipeg, Canadá, quien informó de manera preliminar que la osamenta corresponde a una mujer adulta. 
Aún se está a la espera del informe final sobre este estudio en particular.

Retomando nuevamente el estudio del perfil, en total se extrajeron 7 muestras: 2, de restos óseos humanos, y 5 de sedimentos con conchas de tipo Polymesoda Sólida y de sedimentos con caracoles de tipo, Lissachatina Fulica o Helix Pomatia. Estas fueron obtenidas de los estratos que se encuentran por debajo del depósito donde se hallaron los restos humanos, el propósito era conocer las secuencias cronológicas.

\section{Resultados}

Los resultados de dataciones C-14 permitieron corroborar parcialmente las fechas registradas para la región sur de la costa Caribe nicaragüense. Se ha confirmado que el sitio arqueológico Angie tiene una antigüedad mayor a los 6 mil años (6140 \pm 30 BP/ Beta 375583). de antigüedad. No obstante, se debe recordar que únicamente fueron sometidos a pruebas de C-14, los estratos ubicados a partir del hallazgo de la osamenta humana hasta los $3.55 \mathrm{~m}$ de profundidad a como se muestra en el perfil.

Si la profundidad real del perfil es de $7 \mathrm{~m}$, a como lo expresaron los pobladores de la localidad, entonces, queda pendiente establecer la secuencia cronológica de un área del perfil aproximado de $3.50 \mathrm{~m}$, mediante nuevas dataciones radio carbónicas.

Si tomamos como punto de partida los actuales resultados, probablemente esta tendencia continuará marcándose en los estratos inferiores a los datados y podría significar una mayor antigüedad que la registrada en la década de los 70’s. Queda esta interpretación a manera de reflexión o hipótesis para profundizar en los estudios.

A pesar que los resultados no permitieron determinar la antigüedad de la osamenta humana, se estableció una edad relativa no superior a los 6000 años de antigüedad. Lo anterior se deduce del análisis estratigráfico realizado, donde se observa con claridad que la fosa original de enterramiento se construyó en períodos posteriores a los depósitos estratigráficos datados (ver imagen del perfil).

\section{Perfil oeste de Angie}

Es importante resaltar que de las siete muestras de C-14, cuatro de ellas equivalentes al $57 \%$, registran fechas superiores a los 6 mil años de antigüedad y el $43 \%$ restantes, se ubican entre 5400 y 5830 años

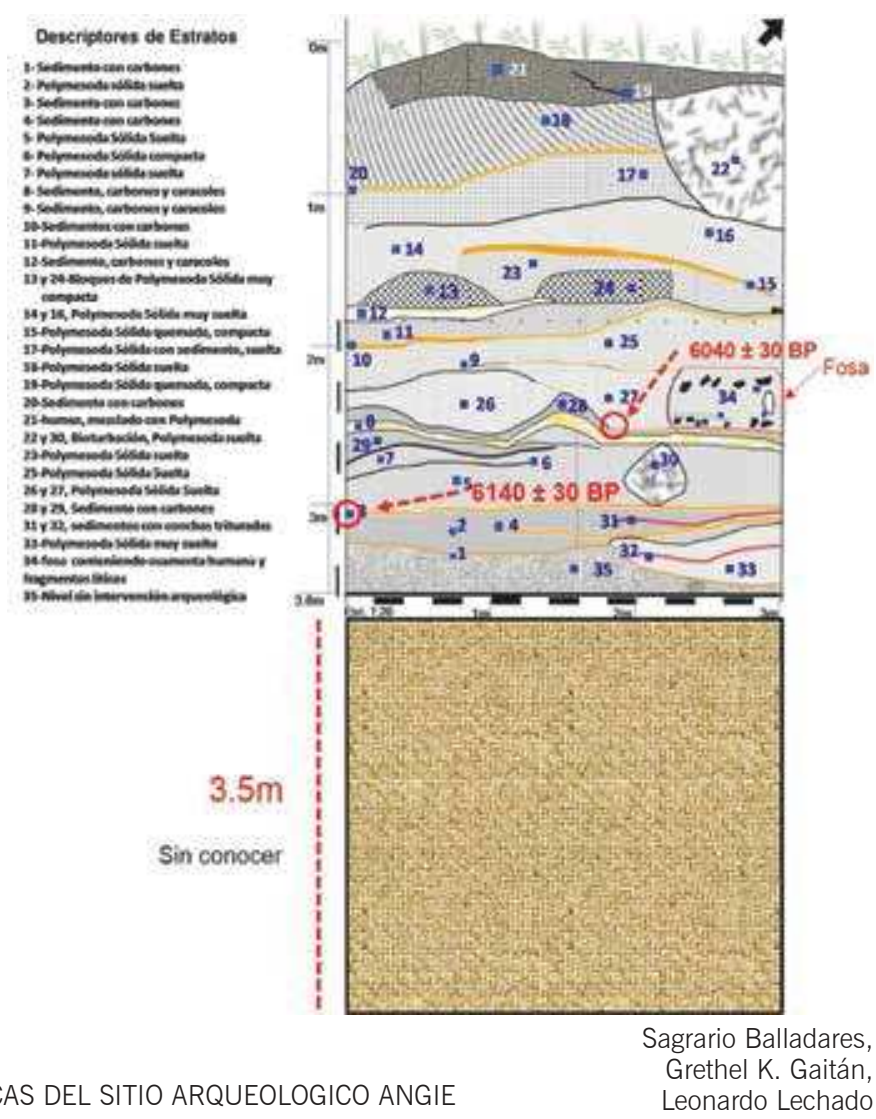

